\title{
Les politiques migratoires en Russie orientale : rôle et influence du «local»
}

Migration Policy in the Russian Far East: Role and Influence of "Local"

Communities

Las políticas migratorias en el este de la Rusia: papel e influencia del «local»

\section{François Dubé}

\section{(2) OpenEdition}

Journals

\section{Édition électronique}

URL : https://journals.openedition.org/remi/6681

DOI : $10.4000 /$ remi.6681

ISSN : $1777-5418$

Éditeur

Université de Poitiers

\section{Édition imprimée}

Date de publication : 1 décembre 2013

Pagination : 133-157

ISBN : 979-10-90426-10-8

ISSN : 0765-0752

Référence électronique

François Dubé, "Les politiques migratoires en Russie orientale : rôle et influence du « local » », Revue européenne des migrations internationales [En ligne], vol. 29 - n4 | 2013, mis en ligne le 01 décembre

2016, consulté le 14 avril 2022. URL : http://journals.openedition.org/remi/6681 ; DOI : https://doi.org/ 10.4000/remi.6681 


\section{Note de recherche}

\section{Les politiques migratoires en Russie orientale : rôle et influence du " local »}

\section{François Dubé ${ }^{1}$}

\section{La périphérie comme lieu de départ de l'analyse}

Aborder l'État russe par sa périphérie, en tenant compte de son isolement par rapport aux processus nationaux et de sa perméabilité aux influences extérieures, constitue une approche particulièrement intéressante. De par son double rapport à l'autre - à la fois au centre et à l'étranger - la périphérie présente diverses caractéristiques susceptibles de nous renseigner aussi bien sur sa condition spécifique d'éloignement que sur les dynamiques de l'État dans son ensemble. À ce titre, l'Extrême-Orient russe (EOR) ${ }^{2}$ se présente comme un terrain de recherche potentiellement très riche pour l'étude des processus migratoires.

En effet, face aux bouleversements qui suivirent l'ouverture de la frontière orientale en 1991, les populations russes des régions avoisinant la Chine durent revoir leurs relations à l'étranger et aux migrants. À la fois enthousiastes, craintives et résignées, celles-ci firent preuve d'une grande polyvalence dans leur confrontation à l'altérité asiatique. L'aspect parfaitement dichotomique de la ligne frontalière - avec ses visas et ses règlements - est en ce sens peu représentatif de l'équivoque constante qu'exprimèrent alors les populations locales dans leur rapport aux migrants et plus particulièrement avec la Chine. Loin de correspondre à l'image des masses xénophobiques que certaines analyses leur prêtent, elles jouèrent ainsi un rôle significatif dans l'adoption des différentes politiques migratoires de la région.

\footnotetext{
1 Candidat au Doctorat, Sciences économiques des minorités ethniques de Chine, Département de Sciences économiques, Université du Ningxia, Yinchuan, Ningxia H.A.R. ; francois.dube.3@ulaval.ca

2 Parmi les nombreuses définitions de I'Extrême-Orient russe, nous retenons celle correspondant au district présidentiel, instauré en 2000, comprenant les districts autonomes juifs, d'Amour, de Saxaline, de Magadan, les Kraï du Kamtchatka, de Xabarovsk, de Primor'e, la République de Saxa et le District autonome de Chukotka. Alors que le sud de ce territoire immense rassemble la grande majorité des habitants (principalement en Primor'e et Xabarovsk), le nord reste peu peuplé et largement déficitaire.
} 
Nous proposons dans cette note de recherche d'explorer plus en détail l'influence exercée par ces populations locales russes ${ }^{3}$ sur la mise en place des politiques migratoires à l'égard de la Chine. Après un aperçu historique du contexte régional, nous procéderons à une présentation des divers mouvements recensés dans la région, selon leurs mobilisations en faveur ou contre l'immigration. Grâce à une série d'entretiens menés auprès d'acteurs directement impliqués à l'époque, nous pourrons ensuite examiner plus en détail la teneur et l'organisation de ces mouvements de mobilisation. Ceci nous amènera à argumenter en faveur d'une révision des constats pessimistes sur la possibilité d'un conflit ethnique en EOR.

Cet examen se bornera dans l'espace à la partie sud de l'Orient russe, soit le Kraï de Primor'e, qui fut au premier plan de cette ouverture régionale, de par les fortes relations économiques et migratoires qu'il entretient avec le Nord-est de la Chine. Nous ciblerons exclusivement la période allant de 1993 à 20004, alors que les difficultés dues à la transition furent les plus importantes et que I'administration du controversé gouverneur Nazdratenko était au pouvoir. En ciblant notre analyse sur l'influence des mobilisations locales, il s'agit ainsi pour nous de restituer à ces communautés leur parole, monopolisée par les élites régionales et moscovites et occultée par les enquêtes précédentes, afin de voir comment celles-ci organisèrent et incarnèrent cette ambivalence : à la fois " rempart " face à l'étranger et " fenêtre sur l'Asie " (Dyatlov, 2009 : 124).

\section{L'Extrême-Orient russe et son voisin}

Longue de 6681 kilomètres, I'actuelle frontière orientale russe fut établie graduellement par l'imposition de différents traités territoriaux à la Chine dans la deuxième moitié du XIXe siècle ${ }^{5}$. Le résultat fut une frontière relativement

3 Nous adoptons le terme de "communautés locales " pour désigner les populations, pour la plupart d'origine slave, arrivées dans la région suite aux mouvements migratoires présoviétique et soviétique. Si le qualificatif de "local " peut sembler quelque peu discutable, notamment du fait que ces colons ne furent pas les premiers habitants de la région, il correspond toutefois à une catégorie mobilisée par les répondants (mestnie, nashi). Cette distinction entre local et étranger se base non pas sur une différence ethnique, mais sur un critère géographique, l'étranger désigne donc autant le Moscovite que le Chinois. Les relations entre ces populations slaves et les peuples autochtones (Evenks, Orotches, Oudegéïs) ou encore avec les populations asiatiques régionales ayant été déportées (notamment les Coréens de Primor'e) engagent des enjeux différents qui méritent un examen particulier et ne seront donc pas abordés dans cette note de recherche.

4 Cette période fut d'abord sélectionnée pour des raisons méthodologiques ; les données relatives à la compilation des journaux étant disponibles dans leur entièreté. Cette période fut également la plus animée en termes de mobilisation et d'activités hostiles à l'immigration. Enfin, elle correspond aux mandats du gouverneur Nazdratenko, un ardent pourfendeur de la politique frontalière de Moscou.

5 La frontière sino-russe, fixée sur l'Argoun par le traité de Nertchinsk de 1689, fut repoussée jusqu'à I'Amour et I'Oussouri en deux temps, par le traité d'Aïgoun en 1858 et le traité de Pékin en 1860, date de la fondation de Vladivostok. Ces derniers furent signés dans des circonstances largement défavorables à l'Empire chinois, alors sous la menace d'interventions étrangères. Suite à l'établissement de la République populaire de Chine en 1949, ces traités furent ouvertement dénoncés comme " inégaux " par Pékin, qui considérait alors y avoir perdu 1,5 million de $\mathrm{km}^{2}$. Exigeant d'abord leur dénonciation, les revendications chinoises devinrent plus précises dans les années 1970. Le gouvernement chinois exigea plutôt des correctifs portant sur l'application du principe de séparation fluviale (touchant 1845 îles) et le transfert d'environ $35000 \mathrm{~km}^{2}$ de terre. Pour une histoire détaillée de I'annexion de l'Extrême-Orient russe par Moscou, voir Stephan (1994: 40-53) et Godron (1995: 168). 


\section{Carte 1 : Carte de la Primor'e}

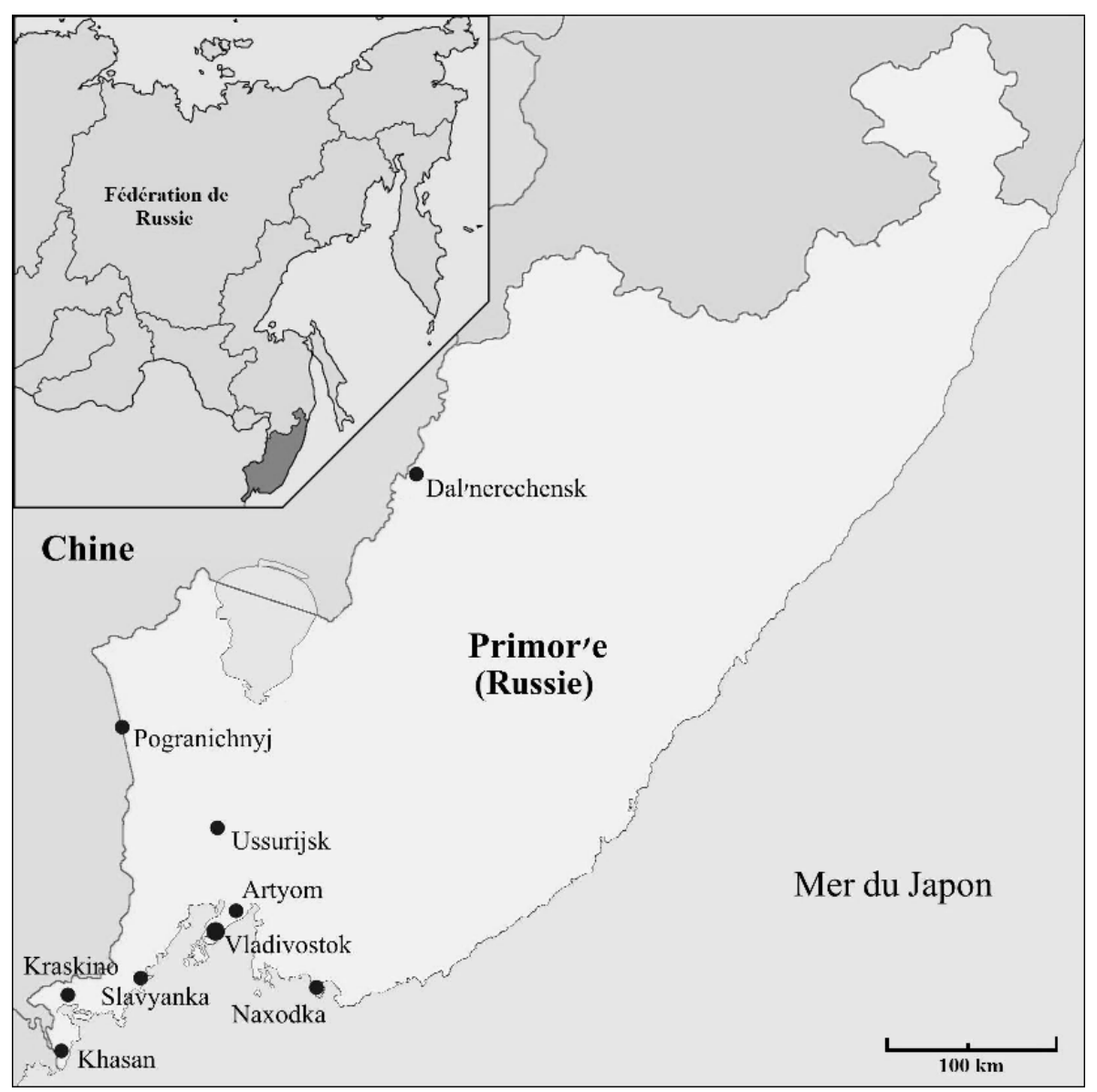

Source : WikiMedia/François Dubé, 2013. 
peu précise, plus ou moins perméable, sans démarcation réelle sur place, donc éminemment fragile. Symboliquement désigné comme russe, le territoire compris dans ces nouvelles limites vit son développement économique retardé et son espace longtemps inoccupé. La population ne s'établit que dans une mince bande le long du fleuve Amour et du Transibérien. L'utilité de la région pour la politique impériale en Asie pouvait alors se résumer dans une triple mission militaire : " défendre la frontière, approvisionner les forces armées et sécuriser les réseaux de communications "(Bliakher et Vasil'eva, $2010: 85)$. En effet, jusqu'à la fin du XIXe siècle, une partie importante des 400000 habitants d'alors était rattachée à la flotte pacifique et à l'armée de terre (Gras et Shvedov, $2010: 129)$.

Malgré ce désintérêt des dirigeants nationaux pour la périphérie orientale, celle-ci occupa une place importante dans la construction géographique de la nation, qui s'articule en Russie dans un positionnement ambigu entre I'Occident et l'Orient. L'appartenance de la Russie à l'Asie et le rôle particulier que I'Extrême-Orient pourrait potentiellement jouer dans cette mission asiatique furent au cœur des réflexions des élites russes au XIXe siècle, et ce, jusqu'à la révolution (Bassin, 2003). Mais ce sont véritablement les avancées du Japon dans la région, notamment la mise en place d'une politique d'occupation et de colonisation de la Mandchourie, qui précipitèrent la prise de conscience des autorités russes sur l'importance stratégique de cette périphérie (Young, 1999). Devant l'éventualité d'une menace militaire concrète, celles-ci mirent en place un programme de colonisation intérieure et de militarisation de la région.

Le nouveau gouvernement soviétique poursuivit la politique tsariste à l'égard de sa périphérie, en raison notamment de l'exacerbation des tensions en Asie. L'objectif prioritaire devint alors sa " transformation en un complexe militaire capable de défendre les frontières, mais aussi de se battre en situation d'isolation du Centre "(Gras et Shvedov, 2010 : 131), qui s'accompagna d'un nouvel élan à la migration interne. Que ce soit lors des affrontements russo-japonais de 1937-1938, des dénonciations des traités inégaux par Pékin et des conflits avec les troupes chinoises en 1969, I'Orient soviétique était alors " entouré de territoires politiquement hostiles et culturellement étrangers " et donc sous "l'épée de Damoclès de la menace étrangère " (Lednev, 2009 : 200). À l'exception de la période de fraternisation soviéto-chinoise, qui dura de la prise du pouvoir par les communistes chinois en 1949 jusqu'à la dénonciation idéologique de I'URSS par Pékin à la fin des années 1950, la frontière fut administrée afin de lutter contre les menaces à l'intégrité territoriale de I'Union soviétique.

\section{Une immigration redoutée, mais nécessaire}

De ce fait, la politique migratoire en Extrême-Orient fut toujours conditionnée par les impératifs du centre, quant au développement de la périphérie, et donc sujet à une contradiction inhérente. À la fois le développement de la région exigeait l'emploi d'une main-d'œuvre chinoise ou coréenne, abondante et disponible, à la fois cette immigration de travail risquait de mettre en péril le projet colonisateur même. Initialement, les politiques impériales furent donc obligées de s'appuyer logistiquement, même temporairement, sur les services de ceux contre qui précisément ces politiques étaient menées. Ce dilemme devint 
fondamental dans la relation qu'entretenait alors la Primor'e aux migrants asiatiques : ceux-ci participent à la consolidation du territoire, mais " soulignent par le fait même la nature conditionnelle de l'hégémonie russe dans la région " (Siegelbaum, $1978: 328$ ).

Cela explique en quelque sorte les incohérences que I'on note dans I'application de cette politique tout au long du XIXe siècle. Ainsi, les paysans chinois établis dans le bassin de I'Ussuri furent expulsés manu militari pour faire place au Transsibérien, alors que près de 10000 ouvriers chinois furent engagés pour construire ce même chemin de fer. On laissa faire le massacre de la population chinoise de Blagoveshchensk par les Cosaques, tout en encourageant cette même immigration vers Vladivostok, afin de contribuer au développement portuaire de la capitale (Dyatlov, 2003). Or, ces épisodes violents ne doivent pas éclipser le fort esprit multiculturel qui caractérisait alors la région. Selon les estimations disponibles, en 1923, les Coréens formaient à eux seuls environ $20 \%$ de la population du sud de la Primor'e et les Chinois plus de 7 \% (Troyakova, 2003). Cette pluralité culturelle et l'interdépendance des colons russes et ukrainiens avec les communautés asiatiques étaient alors un attribut essentiel de la région, qui s'est maintenu jusqu'à la consolidation du régime soviétique.

Car bien que souvent présentés comme des caractéristiques inhérentes du Kraï, I'isolationnisme face au monde asiatique et la "mentalité de forteresse " sont en fait des constructions spécifiquement soviétiques et relativement récentes. L'expulsion de la totalité des communautés coréennes ${ }^{6}$ par les organes de sécurité à l'automne 1937 mit fin à cette tradition d'ouverture et " créa un environnement social russo-centrique hautement artificiel " par rapport aux périodes précédentes (Moltz, 1995 : 513). Dès lors, s'abattit sur la région un rideau de fer qui perdura jusqu'en 1991, date de la réouverture officielle de la ville de Vladivostok aux étrangers.

Avec la dissolution soudaine de I'URSS et la mise en marche de réformes économiques qui affectèrent durement les liens avec la Russie européenne, la réouverture de la frontière devint un facteur décisif dans la vie économique et sociale de la région. La diminution rapide des contrats gouvernementaux en 1991-1992 permit aux provinces chinoises adjacentes (principalement Heilongjiang et Jilin) de pallier ce vide et d'assurer le ravitaillement de la Primor'e en produits de première nécessité (Lukin, 2007 : 292). Ainsi, de 1986 à 1993 , les échanges commerciaux entre les deux pays se multiplièrent par cent et le nombre de passages transfrontaliers atteignit alors un sommet de 1,5 million d'allées et venues (Kireev, 2009 : 10). En 1998, on dénombrait déjà plus de 1600 compagnies des deux côtés de la frontière orientées vers les échanges commerciaux, notamment autour des postes douaniers de Suifenhe-Pogranichnyj et de Hunchun-Kraskino.

Avec ce flux de denrées et de produits asiatiques, la région vit également arriver une quantité non-négligeable de migrants originaires de Chine,

6 Un plan de déportation des populations coréennes fut adopté par Moscou en 1937 dans le but " de lutter contre l'espionnage japonais ". Dans une opération de grande envergure qui devint le premier cas de déportation de masse en Union soviétique, les populations coréennes furent déportées vers les fermes collectives d'Asie centrale, dont environ 100000 au Kazakhstan et 70000 en Ouzbékistan. À ce sujet, voir Kim (2009). 
notamment des trois provinces du Nord-est : Liaoning, Heilongjiang et Jilin (Alexeeva, 2008). Décrite comme un " exode de Chinois opportunistes cherchant à améliorer leur condition économique " (Moltz, $1995: 526)$, ce retour soudain de l'autre, après une absence de soixante ans, put paraître à plusieurs brutal et précipité. Cette inquiétude n'est pas sans lien avec la situation démographique de l'EOR, comptant une population de seulement 6,5 millions (en baisse de 1,5 million depuis 1991), face aux 107 millions des trois provinces chinoises voisines (Kumo, 2010). Ainsi, la pénétration d'une force de travail chinoise, de migrants commerçants et travailleurs, de produits de consommation et potentiellement d'immigrants permanents dans la région devinrent des enjeux majeurs de la mobilisation et de l'action gouvernementale dans les années 1990.

\section{Une transition désorganisée et difficile}

D'abord, il s'avère essentiel de bien situer le contexte particulier du fédéralisme atypique de la Russie des années 1990. Cette variante se caractérisait d'abord par une fragmentation de l'espace économique et politique commun, conséquence directe de la désagrégation du régime soviétique. Le contrôle du Parti communiste de I'URSS, qui exerçait un rôle essentiel de coordination dans les périodes antérieures, ne fut pas entièrement relayé par le nouveau gouvernement démocratique, notamment en périphérie. La fin de ce système signifia donc l'abolition des deux verrous qui assuraient précédemment la cohésion entre les différentes entités fédérées, à savoir la menace de représailles politiques et le contrôle centralisé des ressources (Mendras, $1999: 2$ ).

La répartition des compétences et des pouvoirs entre le centre et la périphérie n'ayant jamais été sérieusement renégociée suite à la chute du système soviétique, ces transformations rapides profitèrent à des groupes qui, occupant déjà des positions de pouvoir, refusèrent de se subordonner aux directives du centre fédéral. On ne peut parler ici de décentralisation, mais plutôt de " désorganisation des politiques centrales ", obligeant les acteurs régionaux à développer leur propre mode de gestion politique et économique (Mendras, 2001 : 7). Il en résulta une nouvelle Russie fédérale à géométrie variable, où chacun tentait de s'assurer une relation privilégiée avec l'appareil présidentiel, tout en maximisant son autonomie locale. La Constitution fut ainsi systématiquement contournée par des relations bilatérales essentiellement personnelles, auxquelles le centre fédéral ne semblait pas pouvoir - ou ne voulait pas - opposer de frein judiciaire et législatif.

Cette situation permit l'émergence et la prise d'autonomie de nouveaux types d'acteurs politiques jusque-là dépendants du centre, qui parvinrent tout au long des années 1990 " à convertir leur capital économique et social en capital politique ", notamment afin d'accéder à la tête des régions (Lallemand, 2008). Ces gouverneurs, désormais élus au suffrage universel, se tournèrent vers les populations et les groupes d'intérêts locaux, où résidait désormais la légitimité de leur pouvoir. L'incapacité du marché à remplacer efficacement l'économie de survie et l'inertie bureaucratique plaça simultanément les populations en situation de dépendance à l'égard des administrations régionales et des grands acteurs industriels (souvent synonymes). Celles-ci furent donc forcées de participer à des politiques clientélistes pour assurer leur survie, accentuant de ce 
fait l'emprise des élites régionales et leur indépendance à l'égard du centre. Or, bien plus que l'apparition d'une "république d'Extrême-Orient séparatiste ", le véritable danger de ce démantèlement de l'État fédéral fut la capture des institutions et des industries régionales par la " criminalité organisée " (Gras et Shvedov, 2010 : 134).

\section{La Primor'e sous Nazdratenko}

Le contrôle des municipalités, des districts et des ressources locales s'avérait donc absolument crucial pour la stabilité et la prise d'autonomie de tels régimes. Chez les dirigeants de I'EOR, ce nouveau clientélisme servit notamment à conforter leur prise de position hostile à l'égard de la politique asiatique de

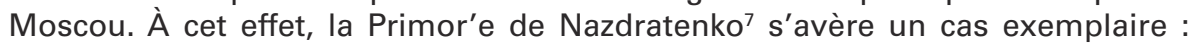
profitant de la désorganisation des agences fédérales, celui-ci put étendre le contrôle de l'exécutif sur l'ensemble de l'appareil d'État. Les contre-pouvoirs se mutèrent alors en " simples institutions supplémentaires pour soutenir le gouverneur "(Tsygankov, 1998 : 342). En monopolisant la quasi-totalité des ressources potentielles, son administration régionale fut capable d'instaurer librement des mesures de contrôle social, tels la manipulation de l'allocation des biens matériels et des pénalités, le renforcement de l'exécutif comme seul porteur de la légitimité, etc.

Cette hypertrophie régionale déboucha sur « des interventions dans les prérogatives du centre fédéral " (Larin, $1998: 8$ ), notamment les politiques frontalières et migratoires. Dans les premières années de la transition, le centre fédéral fut incapable de formuler une législation cohérente, se limitant à la gestion des réfugiés (IOM, $2006: 73)$. Face à la fragmentation du pouvoir exécutif et l'absence de procédures claires pour l'application des lois (Mukomel, $2006: 4$ ), les régions prirent l'initiative de formuler leurs propres politiques migratoires. Celles-ci furent largement conditionnées par les conditions socio-économiques particulières : alors que certaines régions comptaient sur l'immigration pour compenser la baisse de population, d'autres tentaient de limiter et de contrôler I'afflux migratoire (Ryazantsev, 2010 : 1). Bien qu'anticonstitutionnelles, ces mesures furent appliquées par les autorités locales jusqu'en 2001, où la volonté du Kremlin de faire revenir la politique migratoire dans le giron de l'État fédéral se solda par une " sécurisation " de la question migratoire et où la lutte aux illégaux devint la priorité.

Dans un tel contexte, les communautés locales de l'Orient russe, marginalisées sous le précédent système, occupèrent dans la période de transition postsoviétique un double rôle à l'égard des élites régionales, à la fois pourvoyeuses de légitimité et tributaires de leurs ressources. Or, l'influence des communautés locales ne fut pratiquement jamais abordée de manière systématique. Les

7 Nazdratenko fut nommé par le président B. N. Eltsine au poste de gouverneur de la Primor'e le 24 mai 1993. Né en 1949 à Severo-Kuril'ska à Saxalin, il fit ses études et I'essentiel de sa carrière dans le Kraï de Primor'e, contrairement à son prédécesseur Kuznetsov. II fut élu en 1990 en tant que député du parti "Union Industrielle » au Soviet suprême de I'URSS. II est souvent associé à la classe des directeurs rouges, apparatchiks de l'industrie lourde, et affiche ouvertement sa proximité avec le milieu industriel de la région. 
travaux traitant des politiques migratoires en Primor'e s'intéressent plutôt aux aspects plus généraux des relations Vladivostok-Moscou (Larin, 1998 ; Gelbras, 2002 ; Lukin, 1998). D'autres cherchent plutôt à compartimenter le sujet, I'abordant sous le seul aspect des questions territoriales (Iwashita, 2004), du régime politique (Kirkow, 1995) ou de l'intégration économique dans la région de l'AsiePacifique (Rozman, 1997). Seuls les travaux d'Alexseev (2002, 2003, 2004 et 2006) s'inscrivent directement dans l'étude des communautés locales et de la migration chinoise, sans toutefois traiter de leurs interactions avec les autorités régionales ou locales.

D'autres auteurs se sont contentés de présenter une vision essentialiste de la question migratoire, dressant un portrait d'une population nationaliste, xénophobe et naturellement encline à I'affrontement (Stephan, 1994 ; Lockwood, 2001). Ceux-ci insistent particulièrement sur l'incongruité essentielle des cultures slave et asiatique, dont la cohabitation devrait nécessairement aboutir à un affrontement en Primor'e, qualifiée de "Balkans asiatiques " par Nazdratenko (1996: 20) ${ }^{8}$. L'histoire troublée des relations sino-soviétiques nourrirait cette hostilité réciproque, laissant craindre le déferlement (tant de fois annoncé) du péril jaune (Dyachenko, 1996). Cette approche est reprise dans les publications académiques sous la forme d'un catastrophisme migratoire, affirmant, sans aucune enquête sérieuse, la présence de plusieurs millions de Chinois en Extrême-Orient russe seulement (Blank, 1997 ; Shim, 2002).

\section{Replacer le « local » au centre de l'analyse}

Nous proposons ici de sortir de ces conceptions afin de comprendre dans toute leur complexité et leur ambiguïté les diverses formes que prit la mobilisation des communautés locales sur l'enjeu migratoire. Au cœur de notre recherche se situent des groupes dotés d'une capacité et d'une autonomie propre, et non des populations prédéterminées par un antagonisme culturel. Par le fait même, nous délaissons également les approches classiques géopolitiques et diplomatiques au profit d'une conception anthropologique de l'environnement frontalier dans lequel s'articulèrent ces mobilisations (Prescott, 1965). Loin de nous limiter à des pratiques institutionnelles ou à des bornes géographiques, nous mettrons I'accent sur " les processus sociaux, politiques et culturels qui construisent la frontière " et par-delà celle-ci, notre conception de l'autre (Liikanen, 2010).

\footnotetext{
8 L'histoire de la Primor'e est fortement marquée par les affrontements militaires le long de la frontière sino-russe. C'est le cas de la bataille de Khasan en 1938, conséquence de I'occupation militaire de la Mandchourie par le Japon, au cours de laquelle les troupes soviétiques repoussèrent avec succès I'assaut autour de la colline Zaozernaja. Cette bataille devint un symbole de la résistance contre le militarisme japonais dans la région. L'utilisation du territoire chinois comme plate-forme pour lancer des incursions antisoviétiques conditionna en grande partie la politique soviétique à l'égard de sa frontière orientale. Un autre épisode sanglant eut lieu à I'hiver 1969, alors que le différend frontalier entre Pékin et Moscou, qui avait émergé dès 1949, mena à un affrontement armé sur la rivière Oussouri. La bataille eut lieu précisément sur I'île Damanskij, située au centre de la rivière Oussouri qui trace la frontalière sino-soviétique. Après plusieurs mois de provocations mineures, les gardes-frontières chinois auraient donné I'assaut sur l'île sous l'ordre de Pékin, tuant plusieurs gardes-frontières soviétiques. À ce sujet, voir Sapir (1996), Matsusaka (2003), Lee (1983) et Goldstein (2001).
} 
Pour ce faire, nous avons adopté une double approche, s'appuyant à la fois sur une analyse documentaire de la presse régionale et sur une enquête extensive sur le terrain. En tout, 3255 événements furent sélectionnés en fonction de leur pertinence par rapport aux enjeux retenus sur une période de sept ans (1993-2000) dans les journaux locaux, régionaux et nationaux ${ }^{9}$. Parallèlement à cette analyse événementielle, nous avons également mené une importante enquête de terrain, recueillant les témoignages de différents acteurs présents à l'époque. En tout, vingt-quatre entretiens furent réalisés, notamment lors d'un séjour sur place à I'hiver 2011, principalement dans les villes d'Ussurijsk, de Khasan et de Vladivostok. Ces localités furent sélectionnées dans le but de centrer notre enquête sur les principaux foyers d'activités tels qu'identifiés par la revue de littérature et l'analyse événementielle, tout en assurant la représentativité des différents milieux (industriel, urbain, agricole) ${ }^{10}$. À ce croisement de différentes données - témoignages spécifiques et tendances régionales s'ajoute également notre correspondance avec des universitaires et des experts régionaux, qui nous permirent dans un second temps de contre-vérifier nos interprétations.

Cette approche nous permettra d'examiner la qualité des échanges frontaliers et la perception de ceux-ci par les communautés et les autorités locales, plutôt que d'opter pour une quantification de la présence chinoise, souvent trompeuse. Les nombres avancés sur la taille de la présence chinoise en Extrême-Orient russe témoignent d'ailleurs des dérives auxquelles peuvent mener ces analyses : les estimations semblent parfois rivaliser d'exagération, allant de 400000 à 2 millions. Des recherches sérieuses et libres d'intérêts politiques avancent des chiffres plus réalistes et beaucoup moins impressionnants. Même au summum de la saison touristique, les immigrés chinois légaux et illégaux ne seraient en fait que 30000 dans tout le Kraï de Primor'e, soit moins de $1,4 \%$ de la population totale (Gelbras, 2002 : 139). Entre 1996 et 1998, cette proportion aurait varié de 0,3 à 1,1\% (Colin, $2003: 26)$.

Cette méthode possède l'avantage de donner un portrait global des années 1993-2000 et d'en dégager les grandes tendances, à la fois au niveau régional (sujet de la Fédération russe) que local (divisions administratives internes à ce sujet). En ce sens, I'attention accordée aux districts par notre méthode d'analyse

9 Nous avons bénéficié pour ce faire de la collaboration des professeurs M. A. Alexseev de I'Université de San Diego et T. G. Troyakova de I'Université d'Extrême-Orient de Russie (DVGU, Vladivostok), qui nous ont fourni un ensemble de données brutes concernant la presse écrite de la Primor'e. Douze journaux furent recensés ou utilisés : Udarnyj front (Dal'nerechenskij), Sinegorye (Lazovskij), Vibor (Artyom), Naxodinskij Rabochij (Naxodka), Primorets (Xasanskij), Zolotoj Rog (Vladivostok), Novosti (Vladivostok), Utro Rossii (Primor'e), Izvestiya (Russie), Kommersant (Russie), Komsomol'skaya pravda (Vladivostok) et Novaya Gazeta (Russie).

10 Nous avons ciblé les communautés locales et les institutions directement concernées par la politique migratoire, à savoir les commerçants locaux, les militants cosaques, les fonctionnaires municipaux et régionaux, les membres des forces policières et des gardes-frontières. Les répondants furent sélectionnés par l'auteur selon deux critères prédéterminés, à savoir leur représentativité de la diversité des mouvements et des organisations et leur participation aux événements étudiés. Nous avons cherché expressément à multiplier les entretiens tout en variant les caractéristiques des répondants, dans le but d'obtenir un portrait reflétant la pluralité des positions (cadres, militants, observateurs, particuliers), des organisations (privées, publiques), des lieux (marchés, villes, villages) et des postures (hostile ou coopérative). 
événementielle est un apport significatif. En effet, trop souvent les études précédentes ont cherché à accoler à l'ensemble de la Primor'e les qualificatifs typiques de l'Extrême-Orient, devenus clichés à force de répétition (déficit de population, décadence économique, économie basée sur les ressources naturelles). Bien que ceux-ci correspondent à une certaine réalité, ils sont loin de décrire adéquatement la totalité des trente-quatre districts et municipalités du Kraï11. Une telle approche nous permettra de comparer les mouvements favorables et hostiles entre les différents milieux et districts, ainsi que la place des acteurs institutionnels et non-institutionnels à l'intérieur de ceux-ci.

\section{L'impulsion antimigratoire des commerçants locaux}

Le recensement de la mobilisation hostile indique que celle-ci prit des formes variées, dont certaines visaient de manière systématique et soutenue les activités commerciales des migrants asiatiques. Divers regroupements de commerçants locaux, à l'origine de cette mobilisation, vécurent péniblement l'ouverture de la frontière sino-russe, particulièrement dans la période initiale (1993-1997). L'arrivée massive de biens de consommation chinois, accessibles et surabondants, rendit peu attrayants les produits nationaux et locaux. Parallèlement, le rétablissement des flux frontaliers amena des migrants chinois à venir chercher du travail directement dans les marchés et entreprises russes, menaçant les emplois des commerçants et travailleurs locaux. Ce changement brusque dans l'équilibre de la concurrence et l'arrivée soudaine d'une main-d'œuvre étrangère enclenchèrent à cette époque les mouvements de mobilisation hostile.

Afin d'examiner plus en détail ces mouvements, nous avons axé notre enquête sur le district d'Ussurijsk ${ }^{12}$, où cette mobilisation des commerçants locaux prit des proportions inégalées dans le reste de la région, en raison notamment de l'importante communauté de migrants étrangers. Plusieurs commerçants de longue date interrogés dans les marchés gardent en effet des souvenirs précis de cette période, invoquant avec une certaine rancœur l'arrivée

11 Nous pouvons diviser le Kraï de Primor'e en trois sous-ensembles. D’abord, les centres urbains-industriels, c'est-à-dire les trois plus grandes villes : Vladivostok, Artjom et Naxodka. Celles-ci rassemblent près de $45 \%$ des 2 millions d'habitants du Kraï, ainsi que les principaux " points de croissances " de l'économie régionale, essentiellement par leurs industries tertiaire et portuaire (Leonov et Kazanceva, 2006 : 93). Cette zone urbaine-industrielle se caractérise par une présence permanente de migrants chinois, dans les marchés, l'économie de service et l'industrie (portuaire et construction). La deuxième zone est la zone frontalière-agricole, dont l'économie, moins dynamique qu'en centre urbain, est essentiellement agricole et transfrontalière, avec tout de même un centre industriel dans la ville d'Ussurijsk. La présence des migrants chinois y est relativement forte, particulièrement dans l'agriculture et les marchés de production : en 1999, 28 \% des travailleurs chinois enregistrés dans la Primor'e résidaient à Ussurijsk (Colin, 2003 : 25). La dernière catégorie représente plus de $75 \%$ du territoire : I'intérieur des terres et la façade pacifique. II s'agit d'un arrière-pays sous-peuplé, où la présence chinoise et son impact économique sont négligeables. Cela s'explique entre autres par l'économie largement déficitaire de cette région et l'exode de la population vers les zones urbaines dynamiques.

12 Carrefour important sur le trajet du Transsibérien, Ussurisk est situé à soixante kilomètres de la frontière chinoise, sa population s'élève à 160000 habitants et l'économie y est essentiellement agroalimentaire, avec une petite base industrielle. 
des commerçants chinois ${ }^{13}$. Ksenija Hor'kova, par exemple, parle du sentiment de détresse qui affectait alors les commerçants :

"Nous risquions beaucoup à cette époque... notre travail, notre revenu. Bien sûr, nous étions angoissés, énervés... Soudainement, en quelques mois, ce que nous avions bâti pendant des années risquait d'être détruit par l'arrivée des Chinois, [eux qui] ne respectaient pas les lois et les traditions locales".

Le sentiment d'urgence et de panique invoqué dans le témoignage d'Anna Jaroshenko reflète bien le point d'émergence de ces mouvements :

"Nous [les vendeurs du Marché Ussuri] travaillions ensemble, honnêtement, comme une famille, en s'aidant les uns les autres dans cette période difficile, les années 1990. Ils [les migrants] ne pouvaient comprendre notre façon de travailler et de coopérer. [...] Bien sûr, personne n'était content de leur arrivée... Leurs produits sont de mauvaise qualité. Leur façon de travailler n'est pas du tout la nôtre. Nous avons donc tenté de protéger nos commerces".

II transparaît des entretiens que ces mouvements de mobilisation auraient initialement émergé de manière décentralisée, s'appuyant avant tout sur les liens de solidarité que partagent les vendeurs d'un même domaine d'activité ou d'un même lieu de travail. Cette mobilisation s'opérait entièrement sous initiative locale, sans coordination explicite entre les marchés et sans organisation rigide ou soutien externe. À l'intérieur d'un même marché, le mouvement s'articulait autour de quelques personnalités fortes, souvent les vendeurs les plus importants, dégageant autour d'eux un consensus. Les marchés de la région revêtant eux-mêmes un caractère multiethnique, cette mobilisation ne peut être décrite comme s'étant faite sur une base exclusivement ethnico-russe, mais plutôt contre la menace que faisaient peser alors les étrangers sur les locaux ${ }^{14}$.

L'incarnation tangible la plus fréquente que prenait cette mobilisation fut d'empêcher les vendeurs asiatiques d'accéder aux marchés, en bloquant physiquement l'entrée de ceux-ci. Un regroupement de personnes chargées de " surveiller " le marché fut mis en place par les commerçants, de manière tout à fait informelle. V. Ivchenko, par exemple, raconte :

"Nous arrivions tôt, plus tôt qu'eux, moi et quelques autres hommes... Nous nous postions devant l'entrée principale. Lorsqu'ils arrivaient, alors impossible de passer, nous les repoussions... Ils comprenaient, mais quelques fois, il y eut des bagarres, ils sont parfois agressifs. Nous aussi ".

Ne pouvant commercer sur les marchés, certains vendeurs chinois décidèrent de s'installer dans les ruelles à proximité de ceux-ci. Les groupes locaux pouvaient alors se déplacer à l'extérieur du marché pour, selon l'expression utilisée, " nettoyer les alentours " en forçant leur départ.

13 Notre enquête s'effectua dans les deux principaux marchés d'Ussurijsk : Marché central et Marché Ussuri.

14 "Les nôtres " et " les locaux " sont des termes utilisés par K. Hor'kova et par plusieurs autres répondants. 
Des témoignages recueillis auprès des commerçants originaires de Chine confirment ces affrontements initiaux et la difficulté des premiers contacts dans les marchés russes, notamment les expulsions par les vendeurs locaux ${ }^{15}$. Loin de se limiter aux seuls marchés d'Ussurijsk, ces mobilisations hostiles étaient partie intégrante d'une tendance régionale, particulièrement forte en zone rurale. Dès 1993, dans la petite ville agricole de Slavyanka, par exemple, les commerçants locaux s'organisèrent afin d'expulser les migrants asiatiques des marchés et d'interdire la vente des produits à bas prix (Primorets, 06/11/1993). Même phénomène à Dal'nerechensk, pourtant relativement peu touché par la présence chinoise, où les commerçants locaux bloquèrent pendant plusieurs semaines l'entrée du marché agricole aux vendeurs asiatiques (Novosti, 20/08/1993), accusant ceux-ci de détruire les entreprises russes (Utro Rossii, 29/06/1996).

Or, malgré ces mobilisations, plusieurs vendeurs migrants s'établirent tout de même dans les villes, soit directement dans les marchés locaux, soit par la création de leurs propres " marchés chinois " indépendants. La mobilisation hostile des commerçants pouvait prendre alors d'autres formes, visant à ostraciser les vendeurs chinois et à les empêcher de commercer efficacement, dans le but ultime de les faire fuir. Bien qu'ils nient avoir exercé de l'intimidation et fait usage de violence, les vendeurs locaux présents à l'époque affirment avoir pratiqué une " certaine pression " pour empêcher leur établissement permanent dans les marchés. Ceux-ci, par exemple, portaient quotidiennement plainte aux services d'immigration locaux, invectivaient les Chinois et incitaient les clients à ne pas " acheter aux étrangers des produits qui vous rendront malades ". Plus significativement, ils refusaient aux migrants l'accès aux ressources communes du marché (transport des marchandises, sécurité, restaurants, toilettes, services sanitaires, etc.), rendant pénibles leurs opérations commerciales.

\section{Les autorités locales : de cible à soutien des manifestants}

Conjointement à ces actions hostiles, la mobilisation prenait également pour cible les autorités, accusées d'avoir "fermé les yeux " sur l'arrivée des vendeurs migrants dans les marchés. Les pouvoirs locaux et municipaux furent régulièrement invoqués dans les témoignages comme ultimes responsables de cette situation, comme ce fut le cas pour V. Ivchenko :

"En laissant les Chinois vendre leurs affaires sur nos marchés, les pouvoirs nous ont trompés, abandonnés seuls. Nous étions seuls contre eux. Voilà pourquoi nous nous sommes organisés ".

II ressort des témoignages que ces mouvements - incapables d'empêcher à eux seuls l'arrivée des commerçants chinois - cherchaient à cette époque à influencer les autorités locales, en organisant notamment des visites des mairies et des bureaux des élus.

15 Entretien de l'auteur avec Zhangjizhou, quarante-cinq ans, et Qiujun, quarante et un ans, deux commerçants chinois originaires de la ville de Mudanjiang, dans la province du Heilongjiang. Ayant fait partie de la première vague de migrants chinois qui ont traversé la frontière au début des années 1990, ils ont partagé l'évolution de leur relation avec les commerçants locaux jusqu'à aujourd'hui. 
Ces rencontres s'effectuaient d'abord en groupe, sous la forme de marches symboliques, pour ensuite s'organiser plus efficacement, sous la base d'une représentation informelle. Les mêmes commerçants ayant pris naturellement la tête des mouvements étaient alors chargés par les autres de venir discuter avec les autorités. Lors de ces rencontres, qui semblent s'être tenues régulièrement durant les années 1993-1994, ceux-ci présentaient leurs doléances aux autorités locales (des districts et des municipalités), cherchant à influencer leur prise de décision. Ils exigeaient d'eux l'adoption de mesures concrètes afin d'éliminer la présence des vendeurs migrants, tels leur reconduite à la frontière, l'interdiction de commercer, ou dans les cas moins radicaux, le contrôle de la qualité et des prix des produits chinois afin " que tous travaillent juste et honnêtement ".

Selon les témoignages recueillis, il semble que cette mobilisation hostile à l'égard des activités commerciales des migrants ne resta pas sans conséquence sur l'attitude des autorités locales. Comme nous le verrons, ces dernières mirent rapidement en place tout un ensemble de mesures, qui correspondaient essentiellement aux demandes soulevées par les acteurs non-institutionnels. Ce contrôle social hostile prit deux formes principales : alors que certaines mesures visaient à réduire la présence même des migrants asiatiques, afin d'éviter leur installation permanente dans la région et de contrôler leur déplacement, $\mathrm{d}^{\prime}$ autres avaient pour objectifs de répondre aux craintes des corporations locales en s'attaquant particulièrement aux commerçants asiatiques.

Le premier type de contrôle social hostile fut déployé essentiellement par les autorités régionales et ciblait précisément l'immigration illégale. De novembre 1993 jusqu'à la fin de 1994, le parlement de la Primor'e multiplia les résolutions visant un contrôle plus étroit et un resserrement de la surveillance le long de la frontière (Troyakova, $2000: 216)$. Les hautes autorités régionales jouèrent ainsi un rôle majeur dans la réinstauration en janvier 1994 d'un système strict et obligatoire de visa pour les citoyens chinois, ralentissant grandement les flots transfrontaliers (Novosti, 22/07/1994). Dès lors, sous l'ordre du gouverneur Nazdratenko, le service local des gardes-frontières amorça une campagne intensive de lutte contre l'immigration, qui s'incarna concrètement dans tout un ensemble d'opérations policières (les opérations Frontière, Étranger, Régime). Dmitrij Kiseljov, inspecteur senior à la Direction des affaires intérieures de la Primor'e, témoigne :

"Chaque opération s'effectuait à travers le Kraï, dans chaque village et ville où commerçaient des Chinois. Nous contrôlions les documents de chaque Chinois rencontré dans la rue, dans les hôtels, dans les marchés et ailleurs. Si ce Chinois était un touriste ayant délaissé son groupe et refusant de quitter le pays selon le délai fixé par son visa, ou s'il s'adonnait à des activités interdites par son statut de touriste, nous le retenions et le placions dans un autobus, où, sous escorte policière, il était reconduit à un passage frontalier (souvent Grodekovo ou Poltavka). Si son délai de séjour en Russie n'était pas encore expiré, nous lui infligions une amende (en présence d'un traducteur et suivant le protocole) et le prévenions de retourner immédiatement dans son groupe touristique ".

Ces opérations donnèrent lieu le plus souvent à des centaines d'arrestations et de déportations, médiatisées dans un style spectaculaire. En tout, ce sont des milliers d'étrangers qui furent raccompagnés à la frontière pour ne pas avoir respecté la limite de leur visa dans cette période (Kommunar, 25/05/1994). Bien 
que critiquées pour leurs excès médiatiques, il semble que ces opérations s'avérèrent somme toute efficaces : de 1994 à 1998, la proportion de migrants chinois respectant leur délai de séjour passa de $64 \%$ à 99,6 \% (Larin, 2002 : 95). Cette volonté de réduire et de contrôler le flux migratoire resta donc une constante de la politique des organes policiers et régionaux tout au long des années 1990.

Le deuxième type de contrôle social se concentra dans les premières années de l'ouverture frontalière et émana plus particulièrement des pouvoirs locaux et municipaux. Dès 1993, la législature régionale du Kraï de Primor'e, sous la présidence de D. Grigorovich, alloua aux districts d'importantes capacités de contrôle des activités commerciales des travailleurs étrangers (Primorets, 19/10/1993). Les pouvoirs municipaux et les districts ruraux ciblèrent alors l'essentiel de leurs activités de contrôle social non pas sur les migrants en général, mais précisément sur les commerçants chinois. Les autorités locales se firent ainsi l'écho de certains groupements corporatifs qui se mobilisèrent fortement contre la présence des migrants asiatiques dans leur lieu de travail.

Celles-ci multiplièrent alors les résolutions visant à limiter les activités commerciales chinoises. L'exécutif municipal d'Ussurijsk fut à ce titre l'exemple phare de cette collaboration entre les pouvoirs locaux et les mouvements de mobilisation. Les élus municipaux reprirent à leur compte les déclarations hostiles à l'égard des commerçants asiatiques et les demandes des commerçants locaux, en accusant les migrants d'activités illégales, de concurrence déloyale, de criminalité et de violence (Kommunar, 19/02/1994).

Les autorités locales n'hésitèrent pas à joindre à ces déclarations des mesures concrètes. Une réglementation stricte fut adoptée afin de contrôler les activités des Chinois dans les marchés, comme l'interdiction aux vendeurs étrangers d'accéder au Marché central et l'expulsion, le 15 août 1994, des commerçants chinois d'un marché improvisé (Kommunar, 03/09/1994). On adopta également un décret permettant de collecter une taxe sur les citoyens chinois faisant du commerce dans la ville (Kommunar, 17/03/1993). Or, les modalités de collecte n'étant pas explicitées, cette loi rendit quasi-légales la corruption et l'extorsion par les forces policières des vendeurs chinois. Un autre décret obligea chaque migrant chinois à porter dans son passeport un permis spécial avec la signature de son employeur (Kommunar, 11/10/1995). Cette directive rendit possible la reconduite à la frontière immédiate de tout migrant ne possédant pas un emploi certifié, ciblant donc implicitement les commerçants indépendants.

Les fonctionnaires municipaux interrogés confirment ces informations et invoquent la pression qu'exerçaient alors les différentes corporations de vendeurs sur les organes locaux. Selon Aleksandr Egorov, fonctionnaire à la Direction du commerce et de la défense des droits des consommateurs, le conseil municipal d'Ussurijsk était alors inondé de demandes et de griefs contre les activités commerciales des migrants, provenant en grande majorité des commerçants locaux. Ce fonctionnaire rappelle la tension qui régnait dans les marchés et les mobilisations spontanées des commerçants locaux :

"La situation était nouvelle, de grands changements se produisaient dans la ville. [...] Les gens vivaient mal et l'arrivée des Chinois sur les marchés risquait d'empirer la situation des locaux, déjà pas très bonne". 
Les restrictions sur les activités commerciales des migrants étaient alors vues comme une politique justifiée par les circonstances. Les autorités ne manquent pas d'affirmer avoir agi sous la pression des commerçants, afin d'assurer la stabilité et d'éviter des dérives potentiellement plus graves encore.

Il apparaît que les autorités des districts et des municipalités en poste à cette époque se montrèrent particulièrement réceptives aux revendications des mobilisations hostiles. Selon les témoignages, celles-ci agirent sous l'impulsion du groupe local, prenant l'initiative de restreindre fortement les activités commerciales des migrants chinois. Or, comme nous le verrons, cette complicité entre autorités locales et commerçants fut temporaire et ne put s'inscrire durablement dans le temps. Cela doit nuancer la version des faits selon laquelle ces mouvements furent une force déterminante dans la mise en place des restrictions, et non uniquement une opportunité pour les autorités d'investir un domaine d'activités relativement libre de leur influence.

\section{Les milieux agricoles et industriels favorables à I'immigration}

Les mouvements hostiles observés dans les marchés, émanant d'acteurs institutionnels et non-institutionnels, ne s'appliquèrent néanmoins pas aux activités agricoles et industrielles des migrants asiatiques. En effet, contrairement à la situation constatée dans le domaine commercial, les pouvoirs locaux et les employeurs se montrèrent favorables à l'utilisation de la main-d'œuvre chinoise dans certains secteurs ciblés. Nous verrons ici que ces mouvements positifs, d'abord circonscrits aux usines et coopératives rurales, s'étendront graduellement à d'autres sphères, jusqu'à mener à un revirement significatif de leur attitude à l'égard des commerçants chinois.

La mobilisation en faveur de l'immigration chinoise par les acteurs non-institutionnels fut l'initiative quasi-exclusive des employeurs privés des secteurs industriel et agricole. Avec l'ouverture de la frontière, la main-d'œuvre chinoise devint en effet une composante incontournable des usines et des coopératives agricoles en zone rurale. Contrairement aux commerçants chinois, qui furent (dès leur arrivée sur les marchés) la cible des corporations et des autorités locales, l'embauche d'ouvriers étrangers fut encouragée par les entrepreneurs et facilitée par les pouvoirs en place.

Selon nos données, cette tendance, bien que faible initialement, progressa constamment, jusqu'à s'imposer pleinement à la fin de 1999. En zone rurale, d'abord, plusieurs projets significatifs dans le domaine agricole s'appuyèrent sur les travailleurs chinois. Les coopératives des villages de la région de Slavyanka et de Khasan ${ }^{16}$ firent appel de manière significative à la main-d'œuvre chinoise pour relancer et accroître leur production (Primorets, 16/01/1993, 13/01/1994). On observe un phénomène analogue à Dal'nerechensk, où les migrants chinois donnèrent un second souffle à une industrie agricole durement affectée (Udarnyj

16 Situés au sud de la région, Slavyanka et Khasan sont des districts ruraux où l'industrie agricole occupe l'essentiel des activités économiques locales. En proie à un exode des jeunes vers les villes, les sociétés et les fermes sont confrontées à un manque de main-d'œuvre. 
Front, 15/06/1996). L'accès à une main d'œuvre abordable donna alors aux entrepreneurs locaux un avantage concurrentiel important dans une période de sérieux troubles économiques.

Les témoignages recueillis auprès d'acteurs ayant favorisé cette coopération révèlent la facilité avec laquelle celle-ci se mit en place. Loin de se sentir menacés ou victimes de l'ouverture frontalière, ces derniers soulignent ses apports bénéfiques et stabilisateurs pour les entreprises et les communautés locales. Pavel Kornev, responsable de la production dans une coopérative agricole à proximité de Khasan, affirme qu'il eut été difficile d'exploiter son entreprise sans l'aide des migrants à cette époque. Selon son expérience, la coopération s'est faite alors de manière relativement aisée, les autorités locales se montrant compréhensives aux problèmes des entrepreneurs. Contrairement à la situation sur les marchés à la même époque, ceux-ci ne cherchèrent pas à imposer d'obstacles sérieux à l'embauche des travailleurs étrangers dans le domaine agricole.

On observe une situation semblable dans le domaine industriel. En effet, les usines d'Ussurijsk et de Naxodka devinrent des centres importants d'embauche de main-d'œuvre chinoise, avec le soutien ouvert des autorités de ces villes dès 1993 (Kommunar, 13/01/1993). En 1995, on comptait 900 Chinois dans la zone industrielle de la ville portuaire de Naxodka (Zolotoj Rog, 16/01/1996) et en 1998, quelque quarante usines et trente-trois coopératives agricoles employaient plus de 1300 travailleurs chinois à Ussurijsk (Kommunar, 01/12/1998). Leonid Tkachenko, directeur des ressources humaines dans une entreprise industrielle d'Ussurijsk, évoque "l'énorme opportunité " pour les entreprises locales que représentait alors l'ouverture frontalière :

"Avec les réformes, les contrats étaient rares. [...] L'ouverture a permis de rechercher de nouveaux marchés. Et ces travailleurs [étrangers] ont permis de réduire les coûts de production. Tout le monde sait qu'ils sont travailleurs et sérieux ".

\section{Les pouvoirs comme facilitateurs de l'immigration de travail}

Les diverses formes de pression dont furent victimes les commerçants asiatiques ne s'appliquèrent donc pas sérieusement aux travailleurs industriels et agricoles, grâce notamment à une étroite collaboration entre entrepreneurs et autorités. Cette relation assumée et souhaitée dans certains domaines fut en effet catalysée par l'action des acteurs institutionnels, qui utilisèrent différents types de contrôle social à des fins coopératives. Comme nous l'avons vu, celui-ci prit d'abord la forme d'une non-obstruction aux efforts des entrepreneurs industriels et agricoles, et parfois même de coordination active avec ces derniers dans leur recherche de main-d'œuvre. Comme l'indiquent les témoignages des entrepreneurs locaux, certains exécutifs municipaux les assistèrent efficacement, en facilitant le transport et la régularisation des travailleurs asiatiques.

Outre ces efforts de coordination, les autorités n'hésitèrent d'ailleurs pas à recourir directement aux services des migrants chinois dans le domaine de la construction, notamment pour les infrastructures municipales, et ce, dès 1993. Les mêmes autorités qui adoptaient alors des mesures contre les commerçants chinois n'avaient aucune difficulté à confier d'importants travaux d'infrastructure 
publique à des compagnies étrangères, comme ce fut le cas dans plusieurs villes et villages du sud de la Primor'e (Utro Rossii, 21/01/1993 ; Zolotoj Rog, 20/02/1993 et $11 / 08 / 1993$ ).

Les témoignages recueillis auprès des fonctionnaires municipaux en poste à cette époque illustrent bien cette tendance. Nikolaj Musalov de la Direction de la construction municipale, souligne à l'image des entrepreneurs l'inéluctabilité et l'efficacité d'une telle collaboration :

"Ces travaux dans la ville par les Chinois étaient avant tout inévitables. Tout le monde sait qu'ils construisent plus vite, moins cher, et la qualité n'est pas pire que la nôtre. [...]

En fin de compte, personne ne veut faire ce travail, donc cela profitait à tous ".

Questionné sur l'attitude contradictoire de leurs administrations, empêchant les activités commerciales d'une part, mais coopérant eux-mêmes avec les migrants de l'autre, Mihail Shhedrov, membre du conseil communautaire du district sud d'Ussurisk, cherche à rationaliser cette attitude :

"C'était aussi quelque chose comme un test: les changements étaient si rapides à l'époque, on ne pouvait tout de même pas ouvrir la porte et laisser entrer n'importe quoi, n'importe qui. Il fallait d'abord s'assurer de leur honnêteté, de leur qualité, les connaître, eux et leur façon de faire ".

Cette vision de la collaboration entre les pouvoirs locaux et les migrants chinois comme d'une " période de probation ", destinée à préparer le terrain pour une coopération approfondie, est particulièrement éclairante pour expliquer les changements s'étant produits dans la stratégie des autorités. En effet, nous avons vu qu'initialement, le contrôle social des acteurs institutionnels visant à augmenter et à accélérer les flux transfrontaliers se résumait à des domaines précis : I'industrie, l'agriculture et la construction. Or, cette tendance se modifia considérablement à partir de 1997 : la posture coopérative des autorités locales s'étendit graduellement au domaine des activités commerciales, jusque-là entravées par diverses restrictions. Alors que précédemment les pouvoirs tendaient à se faire l'écho des mobilisations hostiles, ils reprirent largement leur autonomie par rapport à celles-ci et réorientèrent leurs politiques vers une coopération accrue avec les commerçants chinois.

Ce changement dans la stratégie des autorités locales et municipales débuta avec la fin des restrictions dans le domaine du commerce, adoptées quelques années auparavant. Ce retournement de situation s'effectua dans plusieurs zones rurales, à des rythmes toutefois différents. À Dal'nerechensk, les pouvoirs favorisèrent I'ouverture de marchés chinois (Utro Rossii, 29/06/1996) alors que les exécutifs des villes frontalières de Pogranichnyj et de Khasan proposèrent la création de zones frontalières de libre-échange économique avec la Chine (Zolotoj Rog, 06/08/1996 ; Utro Rossii, 18/03/1998). En milieu urbain, cette transition vers une plus grande tolérance à l'égard des commerçants asiatiques prit forme plus tardivement. En 1998 et 1999, les maires des trois grands centres industriels de la région, Artyom, Vladivostok et Naxodka, annoncèrent néanmoins leur intention de construire des centres commerciaux chinois (Utro Rossii, 15/05/1998 ; Zolotoj Rog, 13/07/1999). Règle générale, ce désaveu des mobilisations hostiles précédentes fut effectué précisément par les mêmes autorités qui s'en étaient fait les porte-voix en 1993. 
À ce titre, les marchés d'Ussurijsk, lieux d'un intense contrôle social hostile de 1993 à 1996, mettent en lumière la volte-face des autorités locales. Non seulement celles-ci levèrent l'ensemble des mesures excluant les vendeurs chinois, mais elles financèrent et encouragèrent activement l'établissement de ceux-ci. En 1998 et 1999, par exemple, la municipalité prit en charge la construction d'infrastructures dans le " marché chinois" : y furent construits un bureau de poste, un hôtel, un restaurant et des logements, prévus pour abriter plus de 1300 migrants asiatiques (Kommunar, 23/10/1998). La ville alla jusqu'à modifier le réseau local de transports en commun afin de faciliter l'accès à ce marché (Kommunar, 15/01/1998). Celui-ci se vit alors pleinement régularisé, ce qui permit à la ville d'y collecter des taxes de manières officielles, assurant la sécurité des vendeurs et éliminant l'intimidation et la corruption rampante. Sous cette impulsion des autorités locales, les activités commerciales chinoises à Ussurijsk prirent une ampleur inégalée dans la région.

Les fonctionnaires interrogés se montrent d'ailleurs particulièrement fiers de cette réalisation. Le marché chinois d'Ussurijsk est devenu, selon leur expression, " un modèle de coopération commerciale " en EOR et en Russie. Invités à expliquer leur passage brusque d'une posture hostile à une coopération ouverte à l'égard des commerçants chinois, les répondants admettent avoir été " pris de court " lors de la période initiale par les mobilisations hostiles et avoir modifié leur point de vue en élargissant leurs considérations. Alors que les risques d'instabilité et d'affrontements sur les marchés avaient initialement orienté leur posture hostile, comme l'explique Egorov, c'est plutôt l'apport économique des migrants à la communauté qui vint motiver ce revirement :

"Ces commerçants [chinois] sont un facteur essentiel pour la survie des classes les plus pauvres, ils vendent leurs produits à des prix très abordables. II faut seulement en réguler les activités, s'assurer que tout se fasse selon la loi. Ce sont nos citoyens qui en tirent profit ".

Des témoignages du côté des commerçants locaux confirment ce changement dans la position des autorités et également dans l'attitude générale à l'égard des vendeurs chinois par la population. Face à cette impulsion coopérative de l'État et à la popularité toujours croissante des produits chinois, ceux-ci ne purent que prendre en compte le changement de donne. Le choc passé, beaucoup de vendeurs locaux qui se trouvaient à la tête des premières mobilisations prirent alors conscience de l'immuabilité du facteur chinois et cherchèrent à en tirer avantage en l'intégrant à leur stratégie de vente. Dmitrij Kiseljov, ayant directement participé à la mise en place des restrictions à cette époque, témoigne de ce changement, à la fois parmi la population et parmi les commerçants :

" Du côté de nos citoyens, il n'y avait pas de grande joie lorsque nous expulsions les Chinois. Plusieurs étaient mécontents, principalement parce que lors des opérations policières, les "marchés chinois" n'ouvraient pas leurs portes. [...] Dans chaque marché, également, il y avait des "patrons" [xozyain], dont le commerce et les revenus dépendaient du nombre de Chinois sur le marché - ils étaient donc des partisans assumés de la présence des Chinois".

L'émergence de ces "patrons " locaux, tirant profit de la présence des Chinois, montre bien qu'une transition majeure s'effectua à l'époque. Bien que 
l'abandon des mobilisations hostiles ne se fît pas de bon gré pour tous les vendeurs locaux, il ressort que le caractère massif et catégorique du revirement coopératif des autorités semble avoir rendu vaine la poursuite de la mobilisation. Les pouvoirs se montrant désormais imperméables aux demandes des regroupements locaux et la population n'hésitant plus à faire affaire avec les migrants, les commerçants ne purent que "s'accommoder " de la nouvelle situation et donc chercher à collaborer eux-mêmes. Cette posture à l'égard de l'ouverture frontalière s'imposa finalement, si bien qu'aujourd'hui les commerçants locaux, dans leur quasi-totalité, collaborent d'une manière ou d'une autre avec les migrants chinois.

\section{" L'élargissement " des rapports frontaliers}

Selon l'interprétation avancée par Kireev (2009: 4), I'ouverture de la frontière sino-russe à partir des années 1990 amorça la transition de la Primor'e vers une "frontière élargie " (shirokaya granica), notamment par la prédominance des liens transfrontaliers sur les liens internes, surtout dans le domaine économique. Faire le bilan de l'ouverture frontalière, c'est donc constater que les changements majeurs de cette période, malgré leur nouveauté idéologique et leurs objectifs novateurs, " n'aboutirent en fin de compte qu'à une reproduction assez fidèle de la situation du siècle passé " (Kireev, 2009 : 11).

Les conséquences de cette transition, brusque pour certains acteurs locaux, servirent également de moteur aux mobilisations locales. Nous avons vu comment celles-ci ne purent s'inscrire durablement dans le temps. Les commerçants se trouvèrent incapables d'entraver définitivement l'arrivée des migrants sur les marchés, d'autant plus que les populations locales, prises avec des problèmes économiques criants, collaboraient volontiers avec ceux-ci. On observe donc à partir de 1997 une transition progressive - largement favorisée par les autorités - des corporations et des commerçants locaux vers une plus grande acceptation de la présence chinoise sur les marchés. À titre d'exemple, le Marché Ussuri, lieu des premières formes de mobilisation hostile en 1993, rassemblait en septembre 2000 dans un même endroit plus de 700 commerçants russes et 1000 chinois, sans conflits majeurs (Novosti, 25/08/2000).

Selon Troyakova, alors que les autorités tentaient, dans la période initiale, de " maintenir un équilibre entre les campagnes politiques (hostiles) et leurs intérêts économiques ", il apparaît qu'à partir de 1997, la mise en valeur des avantages économiques devint clairement leur priorité ${ }^{17}$. Un incitatif non-négligeable en faveur de ce revirement de position fut les importants bénéfices économiques que les autorités locales espéraient tirer de la régularisation du commerce chinois. Ceci n'est pas sans liens avec la politique budgétaire des transferts fédéraux adoptée en 1994, qui força les administrations locales à trouver de nouvelles sources de revenus afin d'assurer la sauvegarde des services (Leonov et Kazanceva, 2006). Dans ce contexte, les autorités durent revoir leur position sur les flux transfrontaliers, acceptant une régularisation de la présence chinoise orientée vers la résolution d'objectifs économiques (fiscaux), ce qui atténua en grande partie sa sévérité.

17 Entretien avec I'auteur, T. G. Troyakova, 19/08/2010. 
En ce sens, la mobilisation locale semble avoir été prise à son propre jeu. $\mathrm{Ne}$ possédant pas les capacités de réguler eux-mêmes les activités commerciales des migrants asiatiques, les commerçants locaux ont appelé de leurs vœux l'ingérence de l'État dans les marchés. Cette prise de contrôle des activités commerciales par les autorités s'est faite d'abord à l'avantage des groupes locaux et en accord avec leurs revendications. Or, une fois que les pouvoirs eurent affirmé leur capacité à réguler le commerce, les vendeurs locaux ne purent s'opposer lorsque ceux-ci décidèrent en 1997 de modifier leur politique. Les pouvoirs locaux purent s'affranchir facilement de leur agenda précédent et affirmer une nouvelle politique.

\section{Le risque (illusoire) d'un débordement ethnique}

Loin de correspondre au portait peu favorable d'une population motivée par la xénophobie, les mobilisations locales s'exprimèrent dans des tendances contradictoires et dans une constante réévaluation de leurs rapports aux migrants et à leurs activités commerciales. Cette ambiguïté à l'égard de l'ouverture frontalière qui transparaît de nos résultats nous amène à revisiter les pronostics pessimistes et les projections catastrophistes, particulièrement répandus dans le traitement de la région.

Les mobilisations observées en Primor'e furent relayées par certaines figures politiques d'envergure, au niveau fédéral, et réveillèrent des craintes anciennes et des stéréotypes très fortement ancrés dans l'imaginaire russe européen. Perçue comme une preuve de la décomposition de l'État russe, l'arrivée de migrants chinois dans la région fut présentée comme une anomalie et non comme le retour à un mode de coopération transfrontalière existant dans les périodes historiques précédentes.

Notre enquête révèle plutôt une conciliation graduelle entre les bénéfices économiques de l'ouverture frontalière et les peurs d'un déferlement migratoire incontrôlable. II apparaît que les mouvements hostiles initiaux (chez les commerçants locaux, par exemple) peuvent très bien évoluer ultérieurement vers une plus grande collaboration suite à une période d'adaptation. Pour certains acteurs en situation précaire, l'arrivée de l'autre peut même être interprétée comme une opportunité de gains économiques : ce fut le cas pour les cadres industriels et agricoles et pour une majorité de citoyens, voulant profiter de produits à bas prix.

II faut donc relativiser l'ampleur des problèmes transfrontaliers que connut la région et mettre en contraste toutes les ambivalences de la période de transition. Il est alors simpliste de réduire les relations frontalières à une débâcle ou à un " effondrement " (Rozman, 2000). Notre enquête se joint ainsi à d'autres publications récentes, notamment Sullivan et Renz, qui soulignent la présence de tendances régionales précédemment ignorées, notamment " d'une attitude plus pragmatique " à l'égard de la présence chinoise et d'une "frustration " des populations à l'endroit de l'obstruction institutionnelle des initiatives locales de coopération (2010 : 282). Même constat pour Larin (2003), qui fait remarquer que les diverses menaces imputées à la Chine se révélèrent en définitive fictives ou hypothétiques. 


\section{Une région toujours à la recherche de sa place en Asie}

L'installation de Vladimir Poutine au poste de président de la Fédération russe en 2000 et la recentralisation de l'appareil fédéral amorcèrent une nouvelle étape dans le développement du régionalisme russe. Suite à une série de scandales, Nazdratenko fut " démissionné " par le Kremlin en 2001. Par la réaffirmation de l'exclusivité de ses compétences, le centre fédéral sonna la fin des prétentions régionales à influer sur le cours de la politique asiatique de Moscou. L'élection en 2001 de S. M. Darkin au poste de gouverneur de la Primor'e fut d'ailleurs l'occasion d'officialiser en des termes clairs ce changement : " L'EOR a changé d'opinion sur la Chine et ne la considère plus comme une menace ", car elle seule " est en mesure de fournir à la région les ressources ouvrières nécessaires à son développement "(Buszynski, 2006 : 293).

Or, cette transition politique ne semble pas avoir libéré la Russie des contradictions présentes depuis le début de son expansion coloniale dans la région. D'une part, on refuse toujours à la Primor'e d'effectuer une véritable intégration économique avec ces voisins asiatiques, par crainte que celle-ci n'entraîne une perte irrémédiable de souveraineté (Lavrov, 2006). D'autre part, la faiblesse économique du centre fédéral est telle, que la région ne peut non plus se tourner vers le vecteur européen ou opter pour une isolation autarcique (Nikolaev, 2009 : 42). On observe donc la délicate cohabitation d'une "régionalisation par le bas" des populations avec une politique de "souverainisation " par le haut, visant à " limiter l'influence économique des pays voisins à l'Est " (Vidalenc, 2009). Confrontés à cette impasse, les dirigeants fédéraux semblent opter pour un prolongement indéfini de la " mise en suspension ", donnant lieu à une intégration régionale conditionnelle et instable (Bliakher et Vasil'eva, 2010 : 86).

Néanmoins, le règlement des complications engendrées par la dissolution de I'URSS annonce la transition de la région vers une nouvelle ère : le dépassement de I'héritage soviétique (Vardomskij et Golunova, 2002 : 215). Même si cela augure l'arrivée de nouveaux problèmes (narcotrafic, contrebande, déséquilibre commercial), le plus grand danger à la stabilité de la frontière ne se trouve pas aujourd'hui dans de potentielles mobilisations hostiles, mais plutôt dans la présence de tendances contradictoires au sein même des élites dirigeantes. $C^{\prime}$ est cette indécision qui menace de ralentir la coopération transfrontalière des communautés locales et non l'inverse. En ce sens, l'établissement d'une politique migratoire cohérente ne constitue que le début d'un long processus: c'est véritablement la gestion frontalière (border management) qui déterminera la capacité des autorités russes à réconcilier deux objectifs en apparence incompatibles le long de leur frontière asiatique, à savoir " maximiser l'accès aux biens, aux personnes et aux idées désirables, tout en maintenant la sécurité de l’État » (Pratt, 2010 : 4). 


\section{Références bibliographiques}

Alexeeva Olga (2008) Chinese Migration in the Russian Far East: A historical and Sociodemographic Analysis, China Perspective, 3, pp. 21-32.

Alexseev Mikhail (2006) Immigration Phobia and the Security Dilemma: Russia, Europe, and the United States, New York, Cambridge University Press, 286 p.

Alexseev Mikhail (2004) Chinese Migration into Primorskii Krai: Economic Effects and Interethnic Hostility, 21st Century COE Program Slavic Eurasian Studies, 2, pp. 329-365.

Alexseev Mikhail (2003) Economic Valuations and Interethnic Fears: Perceptions of Chinese Migration in the Russian Far East, Journal of Peace Research, 40 (1), pp. 85-102.

Alexseev Mikhail (2002) Chinese migration in the Russian Far East: Security Threats and incentives for Cooperation in Primorskii Krai, in Judith Thorton, Russia's Far East: A Region At Risk, Washington, University of Washington Press, pp. 319-348.

Bassin Mark (2003) Classical Eurasianism and the Geopolitics of Russian Identity, Ab Imperio, 2, p. 257-267.

Blank Stephan (1997) Imperial Decline: Russia's changing role in Asia, London, Duke, $194 \mathrm{p}$.

Bliakher Leonid and Vasil'eva Ljudmila (2010) The Russian Far East in a State of Suspension, Russian Politics and Law, 48 (1), pp. 80-95.

Buszynski Leszek (2006) Oil and territory in Putin's relations with China and Japan, The Pacific Review, 19 (3), pp. 287-303.

Colin Sébastien (2003) Le développement des relations frontalières entre la Chine et la Russie, Les Études du CERI, 96, 45 p.

Dyatlov Viktor (2009) Трансграничные миграции и принимающее общество (Migration transfrontalière et société d'accueil), Ekaterinburg, Édition de l'Université de I'Oural, 396 p.

Dyatlov Viktor (2003) Благовещенская «утопия» (" Utopie » de Blagoveshhensk), Vestnik Evrazii, pp. 123-141.

Garnett Sherman (1998) Russia-China Relations in a Changing Asia, Washington, D.C., Carnegie Endowment for International Peace, 46 p.

Gelbras Vilya (2002) Chinese migration to the Russian far east: A view from Moscow, Monterey, Monterey Institute of International Studies, 16 p.

Godron Anne (1995) Vers le règlement du contentieux frontalier sino-soviétique, Revue d'histoire diplomatique, 2, p. 168.

Goldstein Lyle J. (2001) Return to Zhenbao Island: Who Started Shooting and Why it Matters, The China Quarterly, 168, pp. 985-997.

Gras Cédric et Shvedov Vycheslav (2010) Extrême-Orient russe, une incessante (re)conquête économique, Hérodote, 138, pp. 127-143.

International Organization for Migration (IOM) (2006) Migration Perspectives: Eastern Europe and Central Asia, Vienne, IOM, 147 p. 
Iwashita Akihiro (2004) A 4,000 Kilometer Journey Along the Sino-Russian Border, 21st Century COE Program Slavic Eurasian Studies, 3, 333 p.

Kerr David (1996) Opening and Closing the Sino-Russian Border:Trade, Regional Development and Political Interest, Europe-Asia Studies, 48 (6), pp. 931-957.

Kim German (2009) Ethnic Entrepreneurship of Koreans in the USSR and post Soviet Central Asia, VRF, 445, 91 p.

Kireev Anton (2009), La spécificité de la frontière extrême-orientale de Russie, Oikumena, 2, pp. 70-82.

Kirkow Peter (1995) Regional Warlordism in Russia: The Case of Primorskii Krai, Europe-Asia Studies, 47 (6), pp. 923-947.

Kuminov Andrej (2005) La Chine - La guerre approche, Saint-Pétersbourg, Poligraphie alternative, $353 \mathrm{p}$.

Kumo Kazuhiro (2010) Demographic Situations and Development Programs in the Russian Far East and Zabaikalye, RRC Working Paper Series, 24, 18 p.

Lallemand Jean-Charles (2008) Les hommes d'affaires en politique dans les régions de Russie : émergence, affirmation et déclin d'un type d'acteurs politiques, Politix, 84, $31 \mathrm{p}$.

Larin Aleksander (2003) Даьний Восток России в Восточной Азии (L'Extrême-Orient russe en Asie), Vladivostok, Dal'nauka, $131 \mathrm{p}$.

Larin Aleksander (2002) Посланцы Поднебесной на ДВ - легальные и нелегальные (Les envoyés de la Chine en EOR - légaux et illégaux), Population et société, 69-70.

Larin Aleksander (1998) Китай и Дальний Восток России в Первой Половине 90-х (La Chine et l'EOR dans la première moitié des années 1990), Vladivostok, Dal'nauka, $283 \mathrm{p}$.

Lavrov Serguei (2006) The Rise of Asia and the Eastern Vector of Russia's Foreign Policy, Russia in Global Affairs, 4 (3), pp. 70-77.

Lednev Vadim (2009) L'espace frontalier de I'EOR dans la structure géopolitique sécuritaire de la Russie, MGOU " Histoire et politiques ", 2, pp. 198-202.

Lee Chong-Sik (1983) Revolutionary struggle in Manchuria: Chinese communism and Soviet interest, 1922-1945, Berkeley, University of California, 366 p.

Liikanen Ikka (2010) CurrentTrends in Border Analysis, From Post-Modern Visions to Multi-Scale Study of Bordering, Eurasia Border Review, 1 (1), pp. 17-28.

Lockwood David (2001) Border Economics versus Border Mentality: The Politics of Russia-China Border Trade, Contemporary Europe Research Centre Series, 2, $43 \mathrm{p}$.

Lukin Alexander (2007) Медведь наблюдает за драконом (L'ours guette le dragon), Moscou, Vostok-Zapad, 608 p.

Lukin Alexander (1998) The Image of China in Russian Border Regions, Asian Survey, 38 (9), pp. 821-835.

Matsusaka Yoshihisa Tak (2003) The Making of Japanese Manchuria, 1904-1932, Massachusetts, Harvard University Asia Center, 522 p. 
Mendras Marie (2001) La question de l'État : la recentralisation impossible, Critique internationale, 12, pp. 145-157.

Mendraz Marie (1999) Moscou et les régions : un jeu à qui perd gagne, Critique internationale, 3, pp. 6-13.

Moltz James (1995) Regional Tensions in the Russo-Chinese Rapprochement, Asian Survey, 35 (6), pp. 511-527.

Morris-Suzuki Tessa (1999) Lines in the Snow, Pacific Affairs, 72 (1), pp. 57-77.

Mukomel Vladimir (2006) Immigration and Russian Migration Policy: Debating the Future, Russian analytical digest, 7, $16 \mathrm{p}$.

Nazdratenko Evgeny (1996) Ради наших потомков (Pour notre postérité), Duxoenasledie, 10-12, pp. 81-83.

Nikolaev Mihail (2009) Россия Тихоокеанская (Russie Pacifique), Moscou, Édition du Conseil de la Fédération, 98 p.

Pratt Martin (2010) The Scholar-Practitioner Interface in Boundary Studies, Eurasia Border Review, 1 (1), pp. 29-37.

Prescott Victor (1965) The Geography of Frontiers and Boundaries, London, Hutchinson, $190 \mathrm{p}$.

Rozman Gilbert (2000) Turning Fortresses into Free Trade Zones, in Garnett Sherman, Rapprochement of Rivalry? Russia-China relations in a changing Asia, Lancaster, Carnegie, $430 \mathrm{p}$.

Rozman, Gilbert (1997) The crisis of the RFE, Problems of Post-Communism, 44 (5), 10 p.

Ryazantsev Sergei (2010) The Modern Migration Policy of Russia: Problems and Necessary Directions of Perfection, Workshop on Labor Migrants in Russia and Central Asia, Hitotsubashi University, Center for Economic Institutions, 4.

Sapir Jacques (1996) La Mandchourie oubliée: Grandeur et démesure de l'art de la guerre soviétique, Paris, Éditions du Rocher, 293 p.

Shim Kyong-Wook (2002) La Russie d'Orient à la dérive, Paris, L'Harmatan, 172 p.

Siegelbaum Lewis (1978) Another 'Yellow Peril': Chinese Migrants in the Russian Far East and the Russian Reaction before 1917, Modern Asian Studies, 12 (2), pp. 307-330.

Stephan John (1994) The Russian Far East A History, Stanford, Stanford University Press, 481 p.

Sullivan Jonathan and Bettina Renza (2010) Chinese migration: still the major focus of Russian Far East/Chinese North East relations?, The Pacific Review, 23 (2), pp. 261-285.

Troyakova Tamara (2003) Приморский край (Le Kraï de Primor'e), in ValeryTishkov, Na Puti k Perepisi, Moscou, Aviaizdat, 528 p.

Troyakova Tamara (2000) A View from the Russian far east, in Garnett Sherman Ed., Rapprochement of Rivalry? Russia-China relations in a changing Asia, Lancaster, Carnegie, $430 \mathrm{p}$. 
Tsygankov Andrei (1998) Manifestations of Delegative Democracy in Russian Local Politics, Communist and Post-Communist Studies, 31 (4), pp. 329-344.

Vardomskij Leonid et Golunov Serguei (2002) Безопасность и международное сотрудничество в поясе новых границ России (Sécurité et coopération internationale le long de la ceinture des nouvelles frontières russes), Moscou, NOFMO, 572 p.

Vidalenc Sabrina (2009) L'Extrême-Orient russe : une nouvelle frontière ?, Dossiers du CERI, 2009-9, 7 p.

Wishnick Elizabeth (2002) Chinese migration to the Russian Far East: A Human security Dilemma, Monterey, Monterey Institute of International Studies, $11 \mathrm{p}$.

Young Louise (1999) Japan's Total Empire: Manchuria and the Culture of Wartime Imperialism, Berkeley, University of California Press, pp. 306-411. 